\title{
Comparative Study on Immediate and Delayed Extraction of Mandibular Molars in the Management of Acute Dentoalveolar Abscess
}

\author{
Charles Ezechukwu Anyanechi
}

\begin{abstract}
Lecturer Department of Oral and Maxillofacial Surgery, University of Calabar Teaching Hospital Calabar, Nigeria/ Department of Oral and Maxillofacial Surgery, University of Calabar, Calabar, Nigeria.

ceanyanechi@unical.edu.ng
\end{abstract}

\begin{abstract}
Objective: This study compared treatment outcome between two acute dentoalveolar abscesses in the same patients diagnosed simultaneously in both quadrants of the mandible, treated by either immediate or delayed extractions of the affected first and/or second molars.

Methods: This is a prospective, cohort, clinical-based study. The timing of infected tooth is the predictor variable, whereas the healing parameters are the outcome variables. The outcome variables include timing of extraction, patients' preference of immediate or delayed extraction, healing time of soft tissues surrounding extraction sockets, and development of complication(s). Other variables were grouped into demographic and clinical parameters. Descriptive and bivariate statistics were computed.

Results: The sample composed of 77 patients with 154 molars grouped as follows: control $(n=77,50 \%)$ extracted on first visit and experimental $(n=77,50 \%)$ removed subsequently on the $5^{\text {th }}$ day. There was no significant association between acute dentoalveolar abscess and development of alveolar osteitis $(\mathrm{P}=0.831)$ as complication rates were $5.2 \%$ in control group and $6.5 \%$ in experimental. Most patients preferred immediate extraction, and healing period of soft tissues was 0.5 weeks shorter in the control than experimental group $(\mathrm{P}=$ $0.57)$.
\end{abstract}

Conclusion: The results of this study suggest that in patients with acute dentoalveolar abscess and accessible oral cavity, immediate extraction should be practiced.

Keyword: Comparative, Dentoalveolar abscess, Extraction, Mandible, Molars.

\section{INTRODUCTION}

Acute dentoalveolar abscess is a common clinical presentation in dental surgery and oral and maxillofacial surgery settings across the globe. (1-3) Dentoalveolar abscess describes localized collection of pus in the alveolar bone at the root apex of a tooth. It is the commonest type of dental abscess caused by infection of the pulp chamber and root canal of the tooth, and infections are confined to dentoalveolar tissues. ${ }^{(4,5)}$ The condition which is a serious complication mostly arise from untreated or poorly treated dental caries, facial fractures, periodontal disease, pericoronitis, or failed endodontic treatment or as a consequence of neglected trauma to dentoalveolar tissues. $(6,7)$

The symptoms and signs include pain, swelling and erythema localized to the affected dentoalveolar tissues, although the suppuration can sometimes spread to contiguous structures with or without fatal consequences. ${ }^{(1,2)}$ Other signs are fever, submandibular lymphadenopathy, some degree of trismus, extraoral and intraoral swelling, and affected dentition will be tender to percussion with or without mobility, and patient may be lethargic or extremely sick. ${ }^{(1,6)}$ The presence of trismus in addition to hoarseness of the voice and drooling of saliva should prompt the clinician to an emergency situation. $(8,9)$

The microbiology of acute dentoalveolar abscess is polymicrobial in nature. The introduction of molecular techniques in the isolation of the causative microorganisms has helped researchers to understand the microbial bionomics of dental abscesses. $\left.{ }^{(10,} 11\right)$ Consequently, the increased reliance on molecular techniques has enriched knowledge of the diverse polymicrobial collection that constitutes a dental abscess. (10-13)

Acute dentoalveolar abscess generally remain localized and resolve without complications, if appropriate treatment is administered to a patient who is physiologically immunocompetent. ${ }^{(14)}$ The condition was frequently underestimated in terms of its morbidity and mortality. The possibility of development of complications and the 
associated morbidity and mortality makes it an important public health problem particularly in the study community where late presentation for dental treatment among the populace is a significant factor that determines the clinical course of the condition. (15, 16) Acute dentoalveolar abscess and its complications impose a substantial degree of burden on individuals, communities, and health-care system; hence early diagnosis and appropriate treatment are extremely important.

There has been a controversy regarding the most appropriate approach towards treatment of acute dentoalveolar abscess. While some researchers and clinicians advocate immediate extraction of the offending tooth, others insist on suppression or control of infection with antibiotics and subsequent delayed extraction of the affected dentition, all advocating their practices as the best valid way for preventing local and systemic spread of infection and effect clinical cure. $\left(3,4,7,17,{ }^{18)}\right.$ As a result of this, in contemporary dental practice, there is no consensus among clinicians and researchers over the gold standard management as evidenced by the variety of surgical protocols and prescription of antibiotics. $(17,18)$

Review of the available literature on acute dentoalveolar abscesses suggests comparative studies towards finding the influence of suspected confounding variables on treatment outcome have always been on different patients or in the same patients with different conditions, rather than on the same patients affected by the same condition. The objective of this prospective study is to clinically determine and compare the treatment outcome between two acute dentoalveolar abscesses in the same patients diagnosed simultaneously in both quadrants of the mandible, treated by either immediate or delayed extractions of the affected first and second molars. The null hypothesis of the study was that there is no significant difference between immediate and delayed extraction of mandibular first and second molars involved bilaterally in acute dentoalveolar abscess in terms of patients' preference, healing time of soft tissues surrounding extraction sockets and the complications that will occur during and after treatment.

\section{Methods}

This was a prospective, clinical study involving 77 male and female patients, between the ages of 33 and 72 years who had immediate and delayed extraction of their first and/or second molars in both quadrants of the mandible affected by acute dentoalveolar abscesses under local anesthesia. The pathologic first and/or second molars were those that caused severe toothache, tender soft fluctuant lower jaw facial swelling and submandibular lymphadenopathy, varying degrees of trismus and fever, such that impaired mastication, caused general body discomfort, and kept patient awake at night prior to their clinical evaluation, diagnosis and treatment. In addition, the clinical crowns of the affected molars were either grossly carious, badly broken down by trauma or discolored, and conservatively, was nonrestorable.

For the purpose of this study, the molar extracted on the patients' first visit in the right quadrant of the mandible form the control group, while those in the left quadrant extracted on the $5^{\text {th }}$ day of commencement of treatment and administering antibiotics, clindamycin $150 \mathrm{mg} 12$ hourly for 10days and non-steroidal anti-inflammatory drug (NSAID) medication, diclofenac sodium $550 \mathrm{mg} 12$ hourly for 5 days that lasted for 10 days were the experimental cohort. The study was done at the Oral and Maxillofacial Surgery Clinic of our institution over a period of 7.5 years, from January 2011 to June 2018. This study followed the Declaration of Helsinki on medical protocol and ethics and the Regional Ethics Review Board of the institution approved the study. The inclusion criteria were patients presenting with non-contiguous bilateral acute dentoalveolar abscesses of the mandibular first and/or second molar affecting both quadrants of the mandible simultaneously and were accessible for dental extraction on first visit. Also included in the study were patients that are non-smokers of tobacco including narcotic drugs, not on steroid therapy or having systemic disorders like diabetes mellitus, kidney, hepatic, or myeloproliferative disorders and chemotherapy that would aggravate the condition, interfere with treatment and healing 
process of the extraction sockets. The forceps extraction of each tooth lasted for $10 \mathrm{~min}$.

Bilateral acute dentoalveolar abscesses of the mandibular first and/or second molar associated with lesions, pregnancy, lactating mothers and surgeries that lasted for more than 10 min were not included in the study. Also excluded were cases of chronic, unilateral, and relapsed dento-alveolar abscesses, cases that were inaccessible for extraction on the first visit due to trismus, uncooperative patients, as well as those already using antibiotics because of their symptomatic molars.

Furthermore, abscesses in the two quadrants of the mandible that are contiguous were also excluded in addition to those that involved third molars. The patients' socio-economic class was categorized based on Adedeji's classification. ${ }^{(19)}$ The subjects' oral hygiene status was assessed and graded using a gross plaque scoring method $(+=$ good,$++=$ fair , $+++=$ poor $)$. The size of the abscess was measured intra-orally by the ruler method; using dental floss to take the estimated measurement in the buccal or lingual sulcus depending on the site abscess was greater in dimension. The floss was then placed on a standard ruler procured for this purpose to determine the actual length of the abscess in centimeter (cm). Pus specimens for microscopy, culture and sensitivity were obtained intra-orally by aspiration from the most fluctuant part of the abscess, and processed promptly using anaerobic culture. Before this procedure, the oral mucosa surface to be used for the aspiration was cleaned with a sterile swab. Periapical radiograph of the affected molars, right and left lateral oblique mandibular radiographs were obtained for each patient, assessed for confirmation of the affected molars, and to rule out spread into fascial space(s).

The forceps dental extractions were done by the same surgeon and dental surgery assistant in the same dental surgery setting. In all the patients, these procedures were performed within an interval of 5 days apart for each of the patients from the time the affected first or second mandibular molar on the right quadrant was extracted to the time of extraction of similar dentition on the left quadrant affected by the acute dentoalveolar abscess. When necessary, the extraction sockets were curetted, and pus drained through it. Before treatment, local anesthesia was obtained using $2 \%$ lidocaine with 1: 80,000 adrenaline. The extractions followed standard protocol and all the sockets were irrigated with $0.9 \%$ normal saline solution immediately after extraction.

The duration of surgery, from the time the patient was asked to open the mouth for adaptation of the beak of the forceps round the molar to be extracted and placement of gauze in the socket for the patient to bite on and swallow saliva at the end was recorded in minutes.

All the subjects were given the same postoperative instructions, and prescription; oral non-steroidal anti-inflammatory analgesics (diclofenac sodium $550 \mathrm{mg} 12$ hourly for 5 days) and broad-spectrum antibiotics (Clindamycin $150 \mathrm{mg} 12$ hourly for 10 days). When the second forceps extraction was done on the $5^{\text {th }}$ day after commencement of treatment, patients were advised not to do the warm saline mouth rinse within the next $24 \mathrm{~h}$, but continue thereafter for the next one week. The experimental group sockets whose molars were extracted on the $5^{\text {th }}$ day, and molar sockets of the control group whose pain persist within the sockets beyond the $5^{\text {th }}$ day, such patients were advised to use paracetamol, $1,000 \mathrm{mg} 6$ hourly, pro re nata.

The subjects were reviewed post-operatively in a blinded manner by the same surgeon, (different from the surgeon who carried out the forceps extraction) on the third, and seventh postoperative days. The patients were further reviewed subsequently after $2,4,6,8$ and 12 weeks. The patients' vital signs were measured and documented during these reviews. The primary predictor of acute dentoalveolar abscess was the presence of first and second molars while the outcome variables were occurrence of complications, healing time of soft tissues surrounding extraction sockets and patients' preference of either immediate or delayed extraction of molars.

Complications were diagnosed based on patients' complaints and clinical evaluation during the follow-up period, and was considered as conditions that required further treatment for patient to get well. Similarly, healing is defined as repair of soft tissues of 
the extraction sockets such that the gap is filled by healthy granulation tissues and pain is not felt by the patient if jaw quadrant is put to function. In addition, patients' preference of either immediate or delayed extraction was determined subjectively. The clinical variables recorded were patients' age, gender, socioeconomic class, oral hygiene status, etiology of dentoalveolar abscess, and duration of symptoms before treatment, molars affected, size of swelling, and complaints of the subjects during the postoperative follow-up period. Others were healing time of soft tissues around extraction sockets and patients' preference of either immediate or delayed extraction of molars. The data obtained were documented in a pro forma prepared for the study and analyzed using the statistical package of EPI INFO 7, 0.2.0, 2012 version software (CDC, Atlanta, GA, USA).

\section{Statistical Analysis}

The chi-square $\left(\chi^{2}\right)$ test and Fisher's exact test were used to compare the proportion of descriptive variables between the two groups of acute dentoalveolar abscess, while the Student's t-test compared the means of continuous variables between both groups at a $95 \%$ confidence interval. The values of $\mathrm{P}<0.05$ are considered significant.

\section{RESULTS}

Seventy-seven (77) patients each that had bilateral acute dentoalveolar abscess of the first and second molars which simultaneously affected both quadrants of the mandible were studied. Their ages ranged from 33 to 72 years with a mean age of $52.7 \pm 2.8$ years. Majority $(\mathrm{n}=65,84.5 \%)$ were in the age range of 40 70 years $(\mathrm{P}=0.000$, Table 1$)$. The males outnumber females in all age categories with male: female ratio of $2.3: 1(\mathrm{P}=0.001$, Table 1$)$.

Table 1: Distribution of age and gender of patients with bilateral mandibular acute dentoalveolar abscess.

\begin{tabular}{|c|c|c|c|c|c|c|}
\hline \multirow{2}{*}{ Age Group } & \multicolumn{3}{|c|}{ Gender } & \multicolumn{2}{c|}{ Total } \\
\hline & & \multicolumn{2}{|c|}{ Male } & \multicolumn{2}{|c|}{ Female } & \\
\hline & $\mathrm{n}$ & $\%$ & $\mathrm{n}$ & $\%$ & $\mathrm{n}$ & $\%$ \\
\hline $31-40$ & 5 & 6.5 & 2 & 2.6 & 7 & 9.1 \\
\hline $41-50$ & 21 & 27.1 & 7 & 9.1 & 28 & 36.4 \\
\hline $51-60$ & 15 & 19.5 & 8 & 10.4 & 23 & 29.9 \\
\hline $61-70$ & 9 & 11.7 & 5 & 6.5 & 14 & 18.2 \\
\hline $71-80$ & 4 & 5.2 & 1 & 1.3 & 5 & 6.5 \\
\hline Total & 54 & 70.2 & 23 & 29.9 & 77 & 100.1 \\
\hline
\end{tabular}

Age: $\chi^{2}=224, \mathrm{df}=12, \mathrm{P}=0.000$

Gender: $\chi^{2}=224, \mathrm{df}=12, \mathrm{P}=0.001$

The socioeconomic classes of the patients were as follows: Class I $(n=8,10.4 \%)$, Class II $(n=$ $11,14.3 \%)$, Class III $(n=14,18.2 \%)$, Class IV $(n=19,24.7 \%)$ and Class V $(n=25,32.5 \%)$. The frequency of patients increased as their socioeconomic classes decreased $(\mathrm{P}=0.02)$. The oral hygiene status of the subjects was graded as fair and poor. 
Overall, 154 first and second molars were involved in the abscesses as shown in Table 2. Although more second molars were affected in the experimental group, the distribution of the molars between the study groups showed no significant difference $(\mathrm{P}=0.792$, Table 2$)$.

Table 2: Distribution of molars in both study groups affected by abscess and extracted.

\begin{tabular}{|c|c|c|l|}
\hline Group & First Molar (\%) & Second Molar (\%) & Total (\%) \\
\hline control & $39(25.3)$ & $38(24.7)$ & $77(50.0)$ \\
\hline Experimental & $35(22.7)$ & $42(27.3)$ & $77(50.0)$ \\
\hline Total & $74(48.0)$ & $80(52.0)$ & $154(100.0)$ \\
\hline
\end{tabular}

$\chi^{2}=224, \mathrm{df}=2, \mathrm{P}=0.792$

Table 3: Duration of acute dentoalveolar abscess before treatment commenced.

\begin{tabular}{|c|c|c|l|}
\hline Duration (Days) & Control (\%) & Experimental (\%) & Total (\%) \\
\hline$<4$ & $22(14.3)$ & $20(13.0)$ & $42(27.3)$ \\
\hline $4-7$ & $38(24.7)$ & $39(25.3)$ & $77(50.0)$ \\
\hline $8-11$ & $17(11.0)$ & $18(11.7)$ & $35(22.7)$ \\
\hline Total & $77(50.0)$ & $77(50.0)$ & $154(100.0)$ \\
\hline
\end{tabular}

$\chi^{2}=224, \mathrm{df}=12, \mathrm{P}=0.631$

The duration of symptoms before treatment are shown in table 3 , and suggests no significant difference $(\mathrm{P}=$ $0.631)$ between the two study groups. The etiology of the abscesses was dentalcaries and its sequelae $(n=109$, $70.8 \%)$, failed root canal therapy $(n=23,14.9 \%)$, trauma $(n=14,9.1 \%)$ and periodontal-endodontic lesion $(n=$ 8, 5.2\%). This was statistically significant $(\mathrm{P}=0.01)$ in favor of dental caries and its sequelae. The cases due to trauma were secondary to previously treated mandibular fractures resulting from road traffic accidents. As shown in table 4, the frequency of patients increased with increasing sizes of the abscesses before treatment commenced, although this was insignificant $(\mathrm{P}=0.647)$ when both groups are compared.

Table 4: Sizes of the abscesses before treatment commenced.

\begin{tabular}{|c|c|c|c|}
\hline Size (cm) & Control (\%) & $\begin{array}{c}\text { Experimental } \\
(\%)\end{array}$ & Total (\%) \\
\hline$<2$ & $7(4.5)$ & $8(5.2)$ & $15(9.7)$ \\
\hline $2.1-3.5$ & $16(10.4)$ & $16(10.4)$ & $32(20.8)$ \\
\hline $3.6-5.0$ & $24(15.6)$ & $26(16.9)$ & $50(32.5)$ \\
\hline$>5$ & $30(19.5)$ & $27(17.5)$ & $57(37.0)$ \\
\hline Total & $77(50.0)$ & $77(50.0)$ & $154(100.0)$ \\
\hline
\end{tabular}

$\chi^{2}=224, \mathrm{df}=12, \mathrm{P}=0.647$

The bacteria microorganisms isolated from the abscesses are shown in table $5(\mathrm{P}=0.891)$. They are facultative and strict anaerobes as well as aerobic organisms. The data further shows essentially same micro-organisms were isolated from both groups, but varying degrees of frequency. 
Table 5: Distribution of the bacteria microorganisms isolated from the two groups

\begin{tabular}{|c|c|c|c|c|}
\hline Type of Organism & Control (\%) & Experimental (\%) & Total (\%) & P \\
\hline Streptococcus viridians & $44(12.0)$ & $49(13.4)$ & $93(25.4)$ & 0.891 \\
\hline Klebsiella pneumonia & $39(10.6)$ & $43(11.7)$ & $82(22.3)$ & \\
\hline Peptostreptococcus & $34(9.3)$ & $28(7.6)$ & $62(16.9)$ & \\
\hline Bacteroides specie & $31(8.5)$ & $25(6.8)$ & $56(15.3)$ & \\
\hline Fusobacterium & $18(4.9)$ & $21(5.7)$ & $39(10.6)$ & \\
\hline Streptococcus milleri & $13(3.5)$ & $11(3.0)$ & $24(6.5)$ \\
\hline Saphylococcus aureus & $7(1.9)$ & $4(1.1)$ & $11(3.0)$ & \\
\hline Total & $186(50.7)$ & $181(49.3)$ & $367(100.0)$ & \\
\hline
\end{tabular}

$\chi^{2}=224, \mathrm{df}=12, \mathrm{P}=0.891$

The operating time was found to be 0.3 min longer in the control group (Table 6).

Table 6: Duration of forceps molar extractions between the two study groups

\begin{tabular}{|l|l|l|l|c|c|}
\hline & \multicolumn{5}{|c|}{ Surgery Time (min) } \\
\hline & Mean (SD) & df & t & P Value & Mean difference \\
\hline control & $8.2(1.1)$ & 2 & -0.414 & 0.43 & 0.3 \\
\hline Experimental & $7.9(0.9)$ & & & & \\
\hline
\end{tabular}

The healing time of the 154 extraction sockets is shown in table 7 . The healing period was 0.5 weeks shorter in the control than experimental group $(\mathrm{P}=0.57)$.

Table 7: Healing time of soft tissues surrounding the molar extraction sockets in the two groups.

\begin{tabular}{|l|l|c|c|c|c|}
\hline & \multicolumn{5}{|c|}{ Healing Time (weeks) } \\
\hline & Mean (SD) & df & t & P Value & Mean difference \\
\hline control & $4.7(2.8)$ & 2 & -0.437 & 0.57 & 0.5 \\
\hline Experimental & $5.2(2.6)$ & & & & \\
\hline
\end{tabular}

Also, patients' preference of timing of molar extractions, showed $43(55.8 \%)$ preferred immediate extraction while $34(44.2 \%)$, delayed $(\mathrm{P}=0.35)$. Furthermore, alveolar osteitis was defined as post-extraction clinical condition some patients presented with between three and seven days after extraction, characterized by the feeling of severe pain sometimes worse than the initial toothache, causing inflammation of the alveolar bone, failed blood clot formation or lost totally or partially from the extraction sockets, leaving empty sockets where bone were exposed to the oral cavity. Overall, alveolar osteitis was seen in $7(9.1 \%)$ patients in whom $9(5.8 \%)$ molar extraction sockets were affected. The distribution of the alveolar osteitis in the two groups studied were $4(5.2 \%)$ sockets in $3(3.9 \%)$ patients in the control group whereas in the experimental cohort it was $5(6.5 \%)$ 
extraction sockets in $4(5.2 \%)$ patients which was insignificant $(\mathrm{P}=0.831)$ when the test of significance on the success rate of treatment between the control and experimental groups was compared (Table 8).

Table 8: Test of significance on the success rate of treatment between control and experimental groups in relation to the affected molars.

\begin{tabular}{|l|c|l|c|}
\hline Success rate & Control (\%) & Experimental (\%) & P \\
\hline & $(\mathbf{n = 7 7 )}$ & $(\mathbf{n}=\mathbf{7 7})$ & \\
\hline Complication & $4(5.2)$ & $5(6.5)$ & 0.831 \\
\hline No complication & $73(94.8)$ & $72(93.5)$ & \\
\hline
\end{tabular}

The alveolar osteitis were seen only in males between the ages of 50 and 71 years, those in Class V socioeconomic category who presented between 8 and 11 days after noticing mandibular swelling, sizes of their abscesses greater than $5 \mathrm{~cm}$, second molar extraction sockets, and those patients whose second molars had periodontal-endodontic lesions $(n=4,44.5 \%)$, trauma $(n=3,33.3 \%)$ and failed root canal therapy $(n=2$, $22.2 \%$ ). No micro-organism was isolated from the extraction sockets that developed osteitis. The alveolar osteitis was diagnosed and managed during follow-up when the patients presented between 3 to 7 days postoperatively with these complications. The past medical and social history of patients, carrying out instructions related to the post-extraction did not add to the development of this complication. Treatment was by debridement of the surrounding tissues within the extraction socket to remove necrotic debris and re-establish blood clot. All the patients including those that did not have complications kept to the post-operative appointments and were successfully treated. In addition, certain symptoms that could suggest systemic response to the infections or their hematogenous spread like shivering, lethargy, fatigue and extreme sickness were not found in these patients. Patients' vital signs were as well within accepted physiological range. Also, no patient complained of adverse effect of the drugs prescribed. There was no relapse of the condition, and all the patients were treated on outpatient basis.

\section{DISCUSSION}

This study compared the treatment outcome between two acute dentoalveolar abscesses in the same patients diagnosed simultaneously in both quadrants of the mandible, treated by either immediate or delayed extractions of the affected first and second molars. The success rates of treatment were $94.8 \%$ in control group whereas in the experimental, 93.5\%. Overall, alveolar osteitis was seen in $9.1 \%$ patients in whom $5.8 \%$ second molar extraction sockets were affected in both quadrants of the mandible. The complication rates of extraction sockets were $5.2 \%$ in control group and $6.5 \%$ in the experimental. It will be difficult to compare the findings of this study with earlier reports due to differences in study design.

However, from the available literature which suggests plethora of treatment modalities, there is lack of sufficient evidence to support the superiority of one treatment modality over another. ${ }^{(20,21)}$ Most clinical studies in the management of acute dentoalveolar abscesses are often flawed in design, limiting validity and applicability of results obtained. Some of the studies are inadequately blinded and do not have appropriate inclusion or exclusion criteria in order to control confounding variables. ${ }^{(22,23)}$

Consequently, measurement of the primary outcome of the studies is faulty due to lack of standardization of these variables. Some studies rely on clinical assessment of relatively crude parameters while others utilize both patient responses and clinical examination that are subjective alone rather than being subjective and objective. ${ }^{(24,25)}$ This has resulted in subjects receiving multiple therapeutic regimens which make it difficult to analyze the relative contributions of each intervention to the success of treatment. The present study utilized both subjective and objective clinical assessment for the evaluation of the treatment outcome. However, the abscesses resulted from bacteria and their toxic products in the necrotic pulp chamber

and root canal of the molars breaching into the periapical tissues through the apical foramen and inducing acute inflammation and pus formation, and this 
pathogenesis was documented earlier by Jayadev et al. ${ }^{(3)}$ and Bertossi et al. ${ }^{(16)}$

Although the comparative result obtained between the two groups studied is not statistically significant, and in spite of the duration of surgery lasting slightly longer in the control but with shorter period of healing of soft tissues surrounding extraction sockets, most patients still preferred immediate extraction. Consequently, the outcome with immediate extraction seems better than the delayed extraction group. Isik et al. ${ }^{(26)}$ supports infected teeth be extracted as soon as possible and procedure not to be postponed by giving antibiotics. They observed that if this is done to the contrary, patients use antibiotics with or without prescription which contributes to increased health care spending and formation of antibiotic-resistant bacteria strains.

The proponents of immediate extraction also noted that a necrotic tooth, devoid of blood supply and having necrotic pulp acts as a foreign body and culture medium, the extraction re-established the blood supply as well as provided drainage which relieved pain and pressure enhancing faster clinical and biochemical resolution of the infection and healing potential of the affected tissues. ${ }^{(17)}$ The result of the present study also supports the earlier finding of Johri et al. ${ }^{(18)}$ and Fasalulla et al. ${ }^{(27)}$ the risks of seeding the infection into deeper spaces by performing immediate extraction is low or not tenable but improve patients' overall comfort.

However, some clinicians ${ }^{(28,29)}$ still believe extraction of teeth in the presence of an acute infection may cause microorganisms to seed into fascial spaces causing spread of infection in the host recommending the procedure when infection localizes and the inflammatory responses subsides. This was due to reports ${ }^{(1,2,5,7,9,27,28)}$ about patients developing severe life-threatening complications like septicemia, cavernous sinus thrombosis, brain abscess, mediastinitis, shock, and even death after extraction of acutely infected teeth. These complications were not recorded in this study.

Furthermore for the experimental group, antibiotic therapy initiated soon after diagnosis and before surgery shortens period of infection and minimizes associated risks like bacteremia. Consequently, result of the present study suggests both immediate and delayed extractions in the presence of acute dentoalveolar abscess can be practiced, but better to do immediate extraction. The duration of antibiotics therapy was for 10 days to prevent relapse of the condition. This practice is supported by previous experiences in the study community and informed extracting the molars of the experimental group in the middle of the 10 day dose. ${ }^{(15,29)}$

The etiological factors identified in this study have been reported by other researchers. ${ }^{(1,2,5)}$ The high frequency of dental caries and its sequelae indicates need for community based preventive strategies like encouraging oral health and preventive dental programs, early treatment intervention and dental health education which improves quality of life among the populace in this community, particularly the socioeconomically disadvantaged that are most vulnerable. In addition, it is not rare to have symptomatic hematogenous spread of bacteria in clinically apparently dentoalveolar infection. (6, ${ }^{14)}$ Patients with predisposing factors like diabetes mellitus, cirrhosis of the liver, hepatoma, chronic liver diseases, chronic respiratory conditions, immunosuppression, long-term alcohol and nicotine abuse and poor oral hygiene are likely to have severe spreading infection following acute dentoalveolar abscess. ${ }^{(5,14)}$ In the present study, patients associated with these conditions were excluded, except those with poor oral hygiene. All the patients who developed alveolar osteitis presented with poor oral hygiene.

In the present study, more males presented than females. In an earlier study in this community, males tend to present late for dental treatment leading to minor ailment that requires less invasive treatment to get worse with complications. ${ }^{(15)}$ The male preponderance is similar in most other earlier reports. $(7,17)$ However, more females presented with acute dentoalveolar abscess than males in the cases managed by Akinbami et al. ${ }^{(30)}$ The relationship between acute dentoalveolar abscess and gender may be explained in terms of genetic predisposition and habit of the afflicted patients in relationship to the food they eat and oral hygiene. The mean age obtained

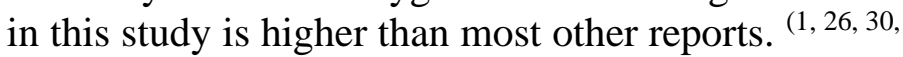
31) This may be due to the study design as the abscesses were bilateral in the same patients rather than unilateral abscess like in most other earlier reports, which is more likely to occur in older patients from the author's clinical experience.

Recent studies $(5,14,18)$ have demonstrated prevalence of more anaerobes than aerobic bacteria in orofacial abscesses particularly when the abscesses have lasted longer periods and cultured under strict anaerobic condition. This is corroborated in the present study. The predominant species associated with acute dentoalveolar abscess include bacteroides, 
fusobacterium, actinomyces, peptostreptococcus, peptococcus, prevotella oralis, prevotella melaninogenica and porphyromas as well as streptococcus viridians which are mostly carbon dioxide dependent, strictly anaerobic Gram positive cocci and anaerobic Gram negative bacilli. (4, 5, 10${ }^{13)}$ The result of this study confirmed the microbiology of acute dentoalveolar abscess is polymicrobial in nature, comprising facultative anaerobes and strict anaerobes with few aerobes, hence the use of broadspectrum empirical antibiotics in the management of the infection. Antibiotics are prescribed to enhance the host immune system's effort to control and eliminate the invading microorganisms. ${ }^{(20,21)}$ Earlier reports, $(9,11,16)$ suggests some acute dentoalveolar abscesses may respond to antibiotics treatment alone, but in the vast majority of cases surgical treatment of the infection like extraction of the offending tooth, curettage of the socket, incision and drainage will be indicated. In the present study, the sockets of the extracted molars provided the drainage channel for the abscesses.

Clindamycin is attractive first line drug in the treatment of acute dentoalveolar abscess. It has favorable activity against Gram-positive microorganisms, including anaerobes and betalactamase producing strains. In low concentration, it is bacteriostatic, but bactericidal activity is achieved clinically with the recommended doses. Gilmore et al. (20) and Von Konow et al. ${ }^{(22)}$ have demonstrated clindamycin's proven clinical efficacy in treating odontogenic infections. The clinical efficacy in this circumstance is based on bacteria susceptibility to it, great oral absorption, less likelihood of bacterial resistance and good concentration in bone, and these benefits were documented by earlier researchers. ${ }^{(20,}$ $22,32,33)$ In addition no patient developed resistance to clindamycin or reacted adversely to it in this study. In fact, it is the drug of choice for patients who are allergic to penicillin and its derivatives.

The main concerns of the dental surgeon in extracting infected teeth are anesthetic failure, dissemination of the infection to contiguous anatomical structures, hematogenous spread, and increased risk of alveolar osteitis. ${ }^{(1,4,5)}$ The advent of new methods and techniques of management of acute dentoalveolar abscess resulted in the reduction of these complications. The only complication recorded was alveolar osteitis which occurred more frequently in the experimental group than the control. This has been reported earlier by other researchers who observed that the presence of an abscessed tooth is a predisposing factor particularly in the mandible area. (26, 27, 32, 34) In addition, the advanced age of the patients, being in lowest socio-economic class who are unlikely to take care of themselves, delayed commencement of treatment and the presence of periodontal-endodontic lesions, trauma and failed root canal therapy coupled with individual variability may have predisposed these patients to developing alveolar osteitis. The alveolar osteitis was not related to any pre-existing periodontal disease or other pathologies within the alveolar bone of the molar extractions' sockets. In addition, very few patients developed osteitis in both quadrants of the mandibular extraction sockets which probably related more to individual variability than to the different treatment options.

The treatment method used to manage the alveolar osteitis has proven efficacy. ${ }^{(35)}$ This complication may be due to patients' and surgeons' factors as documented by earlier researchers. ${ }^{(6,29,34)}$ However, future prospective study will focus on the reason(s) some extraction sockets in immunocompetent patients who have acute dentoalveolar abscess develop complications after tooth extraction.

Oblique lateral mandibular view $\mathrm{x}$-ray rather than panoramic radiograph and/or computed tomography scan was used for the radiological assessment of patients. This was due to their non-availability at the study center during the period of the study. In addition, treatment effects of molar extractions such as patients' post-operative pain level were not used to assess treatment outcome due to oro-facial pain radiation

This study has revealed that acute dento-alveolar abscess emanated mostly from dental caries and its sequelae, those in lower socio-economic classes without good oral hygiene, and the abscess increased in size with delay in treatment worsening clinical outcome. Furthermore, previous comparative studies on acute dentoalveolar abscess have always been on different population of patients, or in the same patients with different clinical conditions, rather than on the same patients affected by the same condition. This study determined and compared the treatment outcome between two acute dentoalveolar abscesses in the same patients diagnosed simultaneously in both quadrants of the mandible, treated by either immediate or delayed extractions of the affected first and second molars with empirical clindamycin and naproxen sodium medication. Although not statistically significant, the outcome obtained with immediate extraction group was better than delayed extraction based on shorter period of healing of soft 
tissues of extraction sockets, patients' preference and fewer alveolar osteitis that developed which generally improved patients' quality of life. However, both immediate and delayed extraction in the presence of acute dentoalveolar abscess can be the clinical practice, but preferably immediate extraction so long as the surgeon can access the oral cavity in the absence of severe trismus.

\section{CONCLUSION}

The outcome with immediate extraction was better than the delayed extraction group based on shorter period of healing of soft tissues of extraction sockets, patients' preference and fewer complications that developed which generally improved patients' wellbeing. However, both immediate and delayed extraction in the presence of acute dentoalveolar abscess can be practiced, but preferably immediate extraction. Future studies will focus on the reason(s) some extraction sockets in immunocompetent patients who have acute dentoalveolar abscess develop complications after tooth extraction.

\section{REFERENCES}

1. Carter L, Starr D. Alarming increase in dental sepsis. $\mathrm{Br}$ Dent J 2006; 200: 243-248.

2. Azodo CC, Chukwumah NM, Ezeja EB. Dentoalveolar abscess among children attending a dental clinic in Nigeria. Odontostomatol Trop 2012; 35: 41-46.

3. Jayadev M, Karunakar P, Vishwanath B, Chinmayi SS, Siddhartha P, Chaitanya B. Knowledge and pattern of antibiotic and non narcotic analgesic prescription for pulpal and periapical pathologies: a survey among dentists. J Clin Diagn Res 2014; 8: 10-14.

4. Külekçi G, Inanç D, Koçak H, Kasapoglu C, Gümrü OZ. Bacteriology of dentoalveolar abscesses in patients who have received empirical antibiotic therapy. Clin Infect Dis 1996; 23: S51-53.

5. Siqueira JF, Rôças IN. Microbiology and treatment of acute apical abscesses. Clin Microbiol Rev 2013; 26: 255-273.

6. Spijkervet FK, Vissink A, Raghoebar GM. The odontogenic abscess. Aetiology, treatment and involvement in the orofacial region. Ned Tijdschr Tandheelkd 2004; 111:120-127.

7. Kuriyama T, Absi EG, Williams DW, Lewis MA. An outcome audit of the treatment of acute dentoalveolar infection: Impact of penicillin resistance. Br Dent J 2005; 198:759-763.

8. Holmberg P, Hellmich T, Homme J. Pediatric sepsis secondary to an occult dental abscess. A case report. J Emerg Med 2017; 52: 744-748.

9. Sawaoka A, Ito T, Yoshida T, Muromachi K, Sakai T, Hamada K, Tani-Ishii N, Ito A. Delayed diagnosis of odontogenic infection: a lesson from altered mental status and abnormal breathing. Oxf Med Case Reports 2018; 11 : OMY 079.

10. Rôças IN, Siqueira JF., Jr. Detection of novel oral species and phylotypes in symptomatic endodontic infections including abscesses. FEMS Microbiol Lett 2005; 250: 279285.

11. Sakamoto M, Rôças IN, Siqueira JF, Jr, Benno Y. Molecular analysis of bacteria in asymptomatic and symptomatic endodontic infections. Oral Microbiol Immunol 2006; 21:112-122.

12. Siqueira JF, Jr, Rôças IN, Souto R, de Uzeda M, Colombo AP. Actinomyces species, streptococci, and enterococcus faecalis in primary root canal infections. J Endod 2002; 28:168-172.

13. Riggio MP, Lennon A. Development of a novel PCR assay for detection of Prevotella oris in clinical specimens. FEMS Microbiol Lett 2007; 276: 123-128.

14. Opitz D, Camerer C, Camerer DM, Raguse JD, Menneking $\mathrm{H}$, Hoffmeister $\mathrm{B}$, et al. Incidence and management of severe odontogenic infections - a retrospective analysis from 2004 to 2011. J Craniomaxillofac Surg 2015; 43: 285-289.

15. Anyanechi CE, Saheeb BD. Reasons underlying failure to seek early dental treatment among patients presenting in a Nigeria Tertiary Hospital. J Med Biomed Res 2013; 12: 3745.

16. Bertossi D, Barone A, Iurlaro A, Marconcini S, De Santis D, Finnoti M, Procacci P. Odontogenic orofacial infections. J Craniofac Surg 2017; 28: 197-202.

17. Igoumenakis D, Giannakopoulos NN, Parara E, Mourouzis C, Rallis G. Effect of causative tooth extraction on clinical and biological parameters of odontogenic infection: A prospective clinical trial. J Oral Maxillofac Surg 2015; 73 : 1254-1258.

18. Johri A, Piecuch JF. Should teeth be extracted immediately in the presence of acute infection? Oral Maxillofac Surg Clin North Am 2011; 23: 507-511.

19. Adedeji GA. Socio-economic and cultural background of hospitalized children in Ilesha, Nigeria. Nig J Paed 1985; 12:111-117.

20. Gilmore WC, Jacobus NV, Gorbach SL, Doku HC, Tally FP. A prospective double-blind evaluation of penicillin versus clindamycin in the treatment of odontogenic infections. J Oral Maxillofac Surg 1988; 46:1065-1070.

21. Adriaenssen CF. Comparison of the efficacy, safety and tolerability of azithromycin and co-amoxiclav in the treatment of acute periapical abscesses. J Int Med Res 1998; 26: 257-265.

22. von Konow L, Köndell PA, Nord CE, Heimdahl A. Clindamycin versus phenoxymethylpenicillin in the treatment of acute orofacial infections. Eur J Clin Microbiol Infect Dis 1992; 11:1129-1135.

23. Fouad AF, Rivera EM, Walton RE. Penicillin as a supplement in resolving the localized acute apical abscess. Oral Surg Oral Med Oral Pathol Oral Radiol Endod 1996; 81:590-595.

24. Paterson SA, Curzon ME. The effect of amoxycillin versus penicillin $\mathrm{V}$ in the treatment of acutely abscessed primary teeth. Br Dent J 1993; 174: 443-449.

25. Lewis MA, Carmichael F, MacFarlane TW, Milligan SG. A randomised trial of co-amoxiclav (Augmentin) versus penicillin $\mathrm{V}$ in the treatment of acute dentoalveolar abscess. Br Dent J 1993; 175: 169-174. 
26. Isik BK, GÜrses G, Menziletoglu D. Acutely infected teeth: to extract or not to extract? Braz Oral Res 2018; 32: e124 $(1-5)$.

27. Fasalulla O, Akhilesh AV, Sobhana CR, Sandhya K, Ravindran V, Sandeep RA. Comparative study of delayed and immediate tooth extraction in the presence of acute infection. Int J Current Res 2017; 9: 55782-55786.

28. Kadaluru UG, Kempraj VM, Muddaiah P. Utilization of oral health care services among adults attending community outreach programs. Indian J Dent Res 2012; 23: 841-842.

29. Anyanechi CE, OG Bassey, FN Chukwuneke, BDO Saheeb. The bacteriology of infected mandibular fractures at the University of Calabar Teaching Hospital, Calabar. Niger J Surg Sci 2010; 20: 6-10.

30. Akinbami BO, Akadiri O, Gbujie DC. Spread of odontogenic infections in Port-Harcourt, Nigeria. J Oral Maxillofac Surg 2010; 68: 2472-2477.
31. Seppänen L, Lemberg KK, Lauhio A, Lindqvist C, Rautemma R. Is dental treatment of an infected tooth a risk factor for locally invasive spread of infections? J Oral Maxillofac Surg 2011; 69: 986-993.

32. Shweta, Prakash SK. Dental abscess: A microbiological review. Dent Res J (Isfahan) 2013; 10: 585-591.

33. Dahlèn G. Microbiology and treatment of dental abscesses and periodontal-endodontic lesions. Periodontol 2000 2002; 28: 206-239.

34. Parthasarathi K, Smith A, Chandu A. Factors affecting incidence of dry socket: a prospective community-based study. J Oral Maxillofac Surg 2011; 69: 1880-1884.

35. Anyanechi CE. Management of alveolar osteitis: a comparative study of two-treatment techniques. J Contemp Dent 2013; 3: 11-14. 